Alonso J.A., López M.J. (2018) Interaction of Hydrogen with Graphitic Surfaces, Clean and Doped with Metal Clusters. In: Andreoni W., Yip S. (eds) Handbook of Materials Modeling. Springer, Cham

\title{
Interaction of Hydrogen with Graphitic Surfaces, Clean and Doped with Metal Clusters
}

\author{
Julio A. Alonso and María J. López \\ Contents

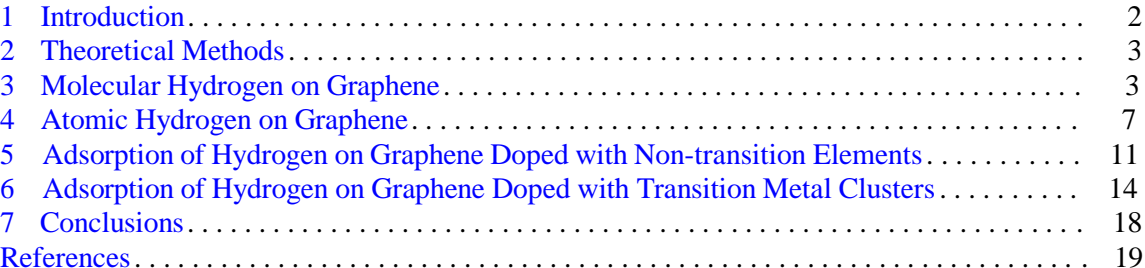

Hydrogen is viewed as a possible alternative to the fossil fuels in transportation. The technology of fuel-cell engines is fully developed, and the outstanding remaining problem is the storage of hydrogen in the vehicle. Porous materials, in which hydrogen is adsorbed on the pore walls, and in particular nanoporous carbons, have been investigated as potential onboard containers. Furthermore, metallic nanoparticles embedded in porous carbons catalyze the dissociation of hydrogen in the anode of the fuel cells. For these reasons the interaction of hydrogen with the surfaces of carbon materials is a topic of high technological interest. Computational modeling and the density functional formalism (DFT) are helping in the task of discovering the basic mechanisms of the interaction of hydrogen with clean and doped carbon surfaces. Planar and curved graphene provide good models for the walls of porous carbons. We first review work on the interaction of molecular and atomic hydrogen with graphene and graphene nanoribbons, and next we address the effects due to the presence of metal clusters on the surface because of the evidence of their role in enhancing hydrogen storage.

J.A. Alonso (M๑) $($ M.J.López

Departamento de Física Teórica, Atómica y Optica, University of Valladolid, Valladolid, Spain e-mail:

jaalonso@fta.uva.es; maria.lopez@fta.uva.es

(C) Springer International Publishing AG, part of Springer Nature 2018

W. Andreoni, S. Yip (eds.), Handbook of Materials Modeling,

https://doi.org/10.1007/978-3-319-50257-1_32-1

\section{Introduction}

The interaction of hydrogen with the surface of carbonaceous materials is a topic of high interest in materials science. Intense work has been motivated by the concern that oil reserves in our planet may be exhausted in five decades or a bit more. Then, hydrogen is viewed as a candidate to replace gasoline as a fuel in cars (Ogden 2002). The engine technology is ready: those cars use an electric motor, based on a fuel cell in which hydrogen coming from the storage tank reacts with atmospheric oxygen producing an electric current, and the only emission from the reaction is water. More specifically, molecular hydrogen is dissociated in the anode of the fuel cell, the protons go through a proton exchange membrane and the electrons through an external circuit creating an electric current, and recombination and reaction with oxygen occurs in the cathode. These novel cars are now fabricated by the major car companies. 
A crucial ingredient in this process is the storage of the hydrogen needed to feed the cell (Jena 2011). Hydrogen is a gas, and the present challenge is to find efficient ways to store enough hydrogen in a tank to be able to run the car for 500 or $600 \mathrm{Km}$. The technology currently in use consists in storing hydrogen gas in steel tanks at high pressures of 350-700 bar. However, in the opinion of many engineers, that method is not the ultimate solution. Hydrogen storage in solid materials is viewed as a safer and convenient alternative. Storage of hydrogen in light solid porous materials is a promising method because the storage mechanisms are the physical or chemical adsorption of hydrogen on the pore walls, and those porous materials show large specific surface areas up to several thousand $\mathrm{m}^{2} / \mathrm{g}$. An interesting class of porous materials is the family of nanoporous carbons. That family includes activated carbons synthesized by physical or chemical activation of carbon-organic precursors or coals (JordáBeneito et al. 2008), carbide-derived carbons synthesized by reacting metal carbides with halogens (Yushin et al. 2006), graphitic nanofibers, and carbon nanotubes. Experiments have been performed to measure the storage capacities of those materials, with the result that the amount of hydrogen stored at low temperatures (around $100 \mathrm{~K}$ ) approaches the onboard storage targets of $6 \%$ of hydrogen in weight (wt) and $40 \mathrm{~g}$ of hydrogen per liter, but the storage capacity is lower at room temperature, far below the storage targets (Ansón et al. 2004; Jordá- Beneito et al. 2007; Lueking et al. 2005; Panella et al. 2005).

Experimental work has shown that the storage capacity of porous carbons can be enhanced by doping these materials with metallic atoms and nanoclusters (Contescu et al. 2009, 2011). Theoretical calculations and computer simulations have been performed to shed light on the mechanisms of interaction between hydrogen and clean or doped porous carbons. In this way, the calculations can help to improve the materials in the quest of the difficult task of fulfilling the storage targets. 